\title{
Serious gaming for the strategic planning process
}

\author{
Adina Aldea, Maria-Eugenia Iacob, Jos van \\ Hillegersberg \\ Centre for Telematics and Information Technology, \\ University of Twente \\ Enschede, The Netherlands \\ \{a.i.aldea, m.e.iacob, j.vanhillegersberg\}@utwente.nl
}

\author{
Dick Quartel, Henry Franken \\ BiZZdesign, \\ Enschede, The Netherlands \\ \{a.aldea, d.quartel, h.franken\}@bizzdesign
}

\begin{abstract}
Serious games have been given more and more attention over the past few years. They are considered to be means of educating, motivating and changing behaviours of participants in a diverse set of domains. This paper proposes a serious game intended for learning how to deal with the strategic planning process of an organisation in a structured way. The game is based on an underlying method which combines well known strategy models in a unique way. The goal of the game is not only to familiarize the participants with the strategy models, but also to emphasize and validate the benefits of the underlying method, such as efficiency and effectiveness, improved communication, better use of organisational resources and capabilities, improved monitoring, and a better overview on the whole strategic planning process.
\end{abstract}

Keywords-gamification, serious gaming, strategic planning process, strategy model, ArchiMate, Balanced Scorecard, Blue Ocean Strategy, Business Case, Business Model Canvas, Dynamic Capabilities, Five Forces Framework, Marketing Mix, PEST analysis, Quantitative Strategic Planning Matrix, Strategy Map, SWOT analysis

\section{INTRODUCTION}

Serious games have received more and more attention over the years and have become increasingly important for education and training. They are used as tools for educating, motivating and changing behaviours in a broad spectrum of domains, such as military, education, government, corporate, healthcare, etc.

One of the main reasons that serious games have become so popular is the fact that they allow participants to experience situations that would otherwise be impossible in the real world due to reasons of safety, cost, time etc. [1]. It is also argued that, with the help of serious games, participants can be guided to develop a specific set of skills. According to [2] game-based learning can help improve performance, increase awareness of the importance of employee roles, can be used for competency testing, assessment, recruitment process, customer \& partner education, as a promotional tool, for induction, as a motivational tool, as an aspirational tool, and for best practices.

This paper introduces a new strategy-based serious game which is being developed by the authors. The target audience for this game is practitioners in organisations which are willing to improve their skills and knowledge about the strategic planning process. In the future, this game can be adapted for educational use, to help students familiarize themselves with strategic thinking.
This game serves two main goals: on the one hand, it helps familiarize the participants with a new way of thinking and working with the strategic planning process, and on the other hand it serves as a demonstration and validation of the underlying method developed by Aldea et al. [3]. The method is a result of a multiple year research that hopes to provide a solution for the Business-IT alignment with the help of strategy models and ArchiMate [30]. When looking at the main pitfalls with strategic alignment there are several aspects that are common in literature, such as an unclear strategy and conflicting priorities, poor communication of strategy, the strategic alignment process is not continuously monitored, strategic goals are not suitable for long-term plans, managers and staff are not involved or recognized in the process, etc. These are some of the issues that the method developed in [3] is trying to overcome and will serve as a main focus and motivation for the serious game. The main contribution of this paper is thus to introduce a new strategy-based game which has its foundation in a well researched method, and to present the results of the first large scale test of the game together with the proposed improvements.

The research methodology that has been used to develop the method is design science as proposed by [4]. As mentioned before, the game serves the role of validation for the method with qualitative and quantitative methods. The paper is structured as follows. The introduction presents the general information about the paper together with its motivation and contribution. Section II includes a review of the relevant literature and section III provides a brief description of the underlying method. In Section IV the game is introduced, with details of its main mechanics, the expected outcomes of the game, the environment of the game organisations, the results of the workshop, and the planned changes to the game. In section $\mathrm{V}$ we discuss about our choice of using students for testing the game and about the current limitations of the game. In the final section (Section VI) we present our conclusions.

\section{BACKGROUND}

Serious games are considered to be formal methods for specifying and planning both education and entertainment content [5] which are supported by a specific game design and mechanics. [7] propose a conceptual framework for designing serious games which includes the following guidelines: identify the skills which need to be developed by the participants, determine what they should learn, determine 
which goals should be achieved, determine the aspects of the game that support learning and engament (incremental learning, linearity of the game activities, short-term memory loads that are placed upon the learner, support given during the game, transfer of learned skills, interaction, learner control, practice, intermittent feedback, rewards, situated and authentic gaming environment, and accommodating the learner's style), identify ways to keep the participants engaged and immersed, offer possibilities for reflection, determine the game genre and game mechanics, and determine a way to score the achievements of the participants. The game mechanics are a vital part of the success of a serious game. They define the structure of the game, the way the participants interract with the game environment, and can impose restrictions (time constraint, limited resources, turns) and offer rewards (recognition, score, achievements) [8].

A mistake that is often made is to consider that incorporating an educational element into a game is enough to make it successful as a serious game, both in terms of fun and educational goals. Similarly, educators and game devlopers assume that learning always occurs in successful games. The true challenge that lies here is finding a trade-off between the two that will not compromise the integrity of one or another. [6] proposes that a successful game should master a balance of three worlds: Reality (no matter how abstract, a game should still have a relation to the real world), Meaning (players should learn something in each game), and Play (a game should be an interactive and engaging tool that immerses people into a fictitious situation).

Based on these guidelines, we can determine that our serious game has a strong relation with Reality (it is based on information from real organisations), has a powerful Meaning (players face the difficulties of the strategic planning process and learn how to deal with them), and has a well-defined universe of Play (succesfully finishing the game relies heavily on the cooperation between participants and on their will to save their organisation).

For designing a powerful game, one more ingredient is needed: the focus of the game [9]. In our case the focus of the game is the strategic planning process. According to [10], [11] and [12] the strategic planning process has six steps: selecting the mission and major goals, analysing the external environment, analysing the internal environment, selecting organisational strategies, implementing the selected strategies, and evaluating the outcomes. For the purpose of the game these steps have been grouped into three categories (analyse, strategize, and implement) which determine the three phases of the game. Based on an extensive literature review of over 35 strategy models, the following were selected for these phases: Business Model Canvas, Business Case, Five Forces Framework, PEST, QSPM, Strategy Map, and SWOT. The underlying method contains a few additional strategy models which will be considered in later iterations of the game.

\section{THE UNDERLYING METHOD}

The Strategy game that is presented in this paper is based on an underlying method which was developed by [3]. The method follows the five steps of the strategic planning process:

visioning process, strategy analysis, strategy formulation, strategy implementation, and strategy evaluation. These five steps are further detailed into nine phases: visioning process, business model, environmental analysis, strategic options, strategic choices, strategy elaborations, measurements \& metrics, strategy implementation, and strategy evaluation. For each of these phases there are a number of strategy models which were chosen as support.

The method also contains two paths that can be followed: the "conventional" path and the "alternative" path. The main difference between these two paths is how the strategy is determined and formulated. The two paths can be chosen based on the experience of an organisation in their market/industry and on the prior information that they possess. For example, an organisation that doesn't have much documented information about their internal and external environment should take the "conventional" path. In the case of an organisation that possesses enough information about their internal and external environment and decides that their current market and/or industry are no longer attractive, the "alternative" path is recommended. Each of the method phases aims at answering a specific question. Figure 1 shows the 9 phases of the method with their activities that will answer the questions.

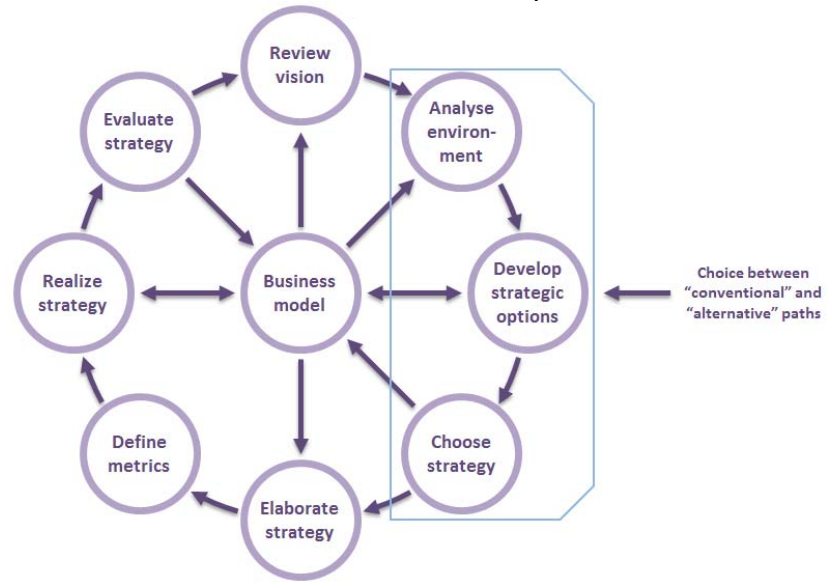

Figure 1 - The underlying method

In the following paragraphs we will shortly present the nine phases of the underlying method. A more detailed presentation of the method can be found in the work of [3].

\section{A. Review vision}

What is the purpose of your organization and what do you want to achieve?

In this phase, the mission, vision and organisational values are defined and/or refined. The method provides certain guidelines on how these three concepts should be handled [12], [13], [14].

\section{B. Business model}

How does your business create, deliver and capture value?

that can aid in this process, of which the Business Mode
The purpose of this phase is to help define or refine the business model of an organisation. There are a number of strategy models 
Canvas was selected, together with the Marketing Mix. The Business Model Canvas (BMC) can be used to define how an organisation creates, delivers and captures value, or how it intends to do so in the future [15] by refining the existing business model. The Marketing Mix can help the organisation to establish their unique selling point by ensuring that their products, people, processes, prices, promotion, etc. are configured to suit the needs to their target market [16].

\section{Anlayse environment}

What forces from the internal and external environment influence your business model?

In this phase, both the external and internal environments of an organisation are analysed in order to determine which factors might influence its performance. Here is where the two paths first come into play. On the one hand, the method proposes several strategy models for the "conventional path": the Resource Based View [17] (resource analysis), the Dynamic Capabilities [18] (capability analysis), the PEST analysis [19] (macro-environment analysis), the Five Forces Framework [20] (industry analysis), and the SWOT analysis [21] (specifies the internal and external factors into strengths, weaknesses, opportunities, and threats). On the other hand, for the "alternative" path, the method suggests using the Blue Ocean Strategy's Six Paths Framework [22] for organisations that want to analyse and determine the commercially compelling blue ocean opportunities.

\section{Develop strategic options}

Which strategic options can help you deal with the environmental influences?

The purpose of this phase is to help organisations determine de options they have based on the results of the environmental analysis, with a continuation of the two aforementioned paths. For the "conventional path" the method proposes using the Confrontation Matrix [23], which helps build alternative strategies based on combinations of strengths, weaknesses, opportunities, and threats. For the "alternative path", the method suggests using the Strategy Canvas (also from the Blue Ocean Strategy), to identify and compare the organisation to the industry based on the main factors that it is competing on.

\section{E. Choose strategy}

Which strategic option should be chosen and replace your current business model?
This is the last phase in which there is a distinction made between the two paths. The goal of this phase is to determine the best course of action for an organisation based on the results from the previous phase. The "conventional" path uses the Quantitative Strategic Planning Matrix [24] as a tool for choosing the best strategy based on how in line it is with the strengths, weaknesses, opportunities, and threats of the organisation. The "alternative" path is focused on reconfiguring the value curve of the organisation based on the factors that the industry competes on, with the help of the Four Actions Framework (also from the Blue Ocean Strategy).

\section{F. Elaborate strategy}

How can you elaborate the chosen strategy?
Here is where the choices of the organisation will be specified into achievable objectives. The

strategy model chosen for this is the Strategy Map, which together with the Balanced Scorecard can provide an all-round solution towards strategy implementation. The Strategy Map [25] proposes determining four layers of objectives which should have causal relationships: financial, customer, internal processes, and learning and growth objectives.

\section{G. Define metrics}

How can you measure and monitor the implementation of your chosen strategy?
The objectives which are determined in the previous phase are translated into traceable and implementable actions. As mentioned before, the strategy model chosen for this is the Balanced Scorecard [26], which to help determine the measures that will be used to evaluate the chosen objectives, the targets that need to be met after implementation, and also the initiatives that need to be undertaken by the organisation in order to fulfil these objectives.

\section{H. Realize strategy}

How can you implement the chosen strategy?

This phase is focused on translating the chosen strategy and organisational goals into efficient and effective organisational change. Because the method is designed to have a connection to Enterprise Architecture, the ArchiMate modelling language [27] will be used for this task in collaboration with the Business Case [28]. The Business Case can be used to evaluate the costs and benefits of all the projects that have been modelled with ArchiMate.

\section{Evaluate strategy}

How can you evaluate the alignment between the

expected and realized results of the implementation?

This final phase focuses on evaluating the progress and results of the strategy implementation. The method proposes using the Balanced Scorecard for this task as well since the objectives, targets and initiatives have been determined with this strategy model.

The method is still being developed and will be further evaluated and validated throughout the following years. One of the ways chosen to validate the method is through the serious game presented in this paper.

\section{THE GAME}

The game is designed to be a short demonstration of the underlying method and can be used as a means for validation. The participants can use the game to easily familiarize themselves with the method and to experience some of its benefits.

The game is designed to be a collaborative experience in which participants have at their disposal a set of cards, tasks they need to complete, a cheat sheet with information about the strategy models and some additional materials.

The game is intended to be played with three teams, each with 2-5 players. The three teams will collaborate with each other in a unique manner that will simulate the way 
information is transferred and used in an organisation. Each of the three teams will represent a travel agency that is part of the same market. The three agencies are experiencing similar problems with their external environment that determines them to adjust their strategy. The internal environment of the three agencies is similar but is governed by one major difference, namely the way they have built their competitive position. Based on the strategy model of [29], the three agencies can be identified as pursuing Product leadership, Customer intimacy, and Operational excellence. More details about the three agencies can be found in sub-section B - The travel agencies.

The game is structured in three main phases that are based on the underlying method: strategize, elaborate, and implement. Each of these phases has a number of steps that need to be followed. Iterations can be done at any point in the game.

Analyse: In this phase the participants will analyse the current internal and external environments of their organisation.

Strategize: This phase will guide participants through the process of formulating alternative strategies based on the circumstances of your internal and external environments. Also they will gain insights into how to make the best decision regarding what strategy would be the most advantageous for their organization by obtaining and maintaining competitive advantage.

Implement: This phase will guide participants through the process of transforming the chosen organisational strategy and objectives into implementable projects. Each of the projects have a given cost that needs to be taken into account when selecting which ones to implement in order to make the best use of the limited resources that the organisation has at its disposal. This phase contains only one step, namely Strategy implementation.

\section{A. Game mechanics}

As mentioned before, the three teams will represent three travel agencies that are competing in the same market. The main aspect regarding the mechanics of the game is that it is intended to be played cooperatively. This choice was made in order to also portray the benefit of improved communication that the underlying method provides. Besides improved communication within an organisation, several other benefits will be experienced by participants, such as: improved decision making, an overview at all times of the strategic planning and implementation processes, better planning, improved allocation of resources, and the usefulness of having all the relevant information in one place.

The competitive aspect of the game comes into play after the completion of each phase. Let's say that there are three teams (team A, B and C) and three agencies (agency X, Y and $Z$ ). Team A represents at the start of the game agency $X$, team $\mathrm{B}$ represents agency $\mathrm{Y}$, and team $\mathrm{C}$ represents agency $\mathrm{Z}$. After the first phase ends, the teams will change the agencies that they represent with each other. That would mean that team A now represents agency $\mathrm{Y}$, team $\mathrm{B}$ represents agency $\mathrm{Z}$, and team $\mathrm{C}$ represents agency $\mathrm{X}$. Now each team is faced with a situation in which they need to elaborate on a strategy that they did not choose for and don't have any extra information about the reasoning that went behind that decision other than what is written down by the other team. This simulates what happens in most organisations. The top management makes decisions about the strategy that the organisation should pursue and gives guidelines about that to the middle management. The middle management needs to follow up on that strategy even if they didn't participate in the decision making process.

Another aspect that is introduced in the game is an unexpected event. This event will occur after the completion of phase 2. By introducing this aspect in the game, the teams will be forced to adjust their strategy and chosen objectives in order to adapt quickly to the new situation. This will mean going through phase 1 and 2 at a quicker pace, which is possible to the fact that since they are already familiar with them. After the challenge of the event is completed, the participants can proceed to phase 3 and choose the relevant projects for the adjusted strategy.

A final aspect of the game mechanics concerns the duration of the game. In order to keep the length of the game relatively small, the amount of information the participants will work with is limited. For example, they will be given the option of having a new office in one of three predefined locations, each with their benefits and risks. Also there will be three new destinations that the teams can choose to offer to their customers. These will also have their own benefits and risks, which will make all the choices balanced. Another way to limit the length of the game was to provide the first three steps of the first phase completely filled in. The participants will not have to fill in anything for these steps but they will need to use the information that is provided there in the following steps.

\section{B. The game scenario}

There are three main competitors in the market that divide between them equally the majority of the market share: Travel Co., JourneyTime, and Tourism Inc. All three agencies have been experiencing problems since the financial crisis started. In order to be able to remain competitive in the current market, the travel agencies will need to try to adjust their strategies.

Current competitive strategies:

- Travel Co.: complementary products and services (Product leadership)

- JourneyTime: excellent customer service and support (Customer intimacy)

- Tourism Inc.: online purchase and payment of tickets (Operational excellence)

The introduction of an element such as the financial crisis allows us to set a realistic problem that most organisations are facing nowadays. Based on this external factor we can introduce realistic concerns that organisations might have, such as diminishing revenues because of less customers. We use this to set up the problem that the participants need to overcome, thus the goal of the game.

External Environment 
Besides the financial crisis, which has been the major determinant for the problems the three agencies have been experiencing lately, there are also other factors that influence them negatively. One of the main concerns of most travel agencies is the removal of the commissions offered by the airlines. This would mean that the margin of the agencies would be severely affected since a majority of them rely almost fully on these types of commissions. Also some major threats to each of the agencies are the main strengths of the other agencies. As an example, Travel Co. has as a main strength the all-inclusive travel packages that it offers, thus the excellent customer service and support offered by JourneyTime, and the option to purchase tickets online offered by Tourism Inc. are major threats to Travel Co. One consequence of the financial crisis is that airlines are reducing the fares they are offering for the existing routes. Thus agencies have fewer seats that they could offer to their customers and less options for a choice of time and date. Another consequence of the financial crisis is that the travel market is stagnating at the moment and because of that the customer loyalty has decreased since their power of negotiation has increased relatively to the agencies. If the offering of an agency doesn't fully satisfy their needs, they will easier switch to another agency that might have a better offer at the moment.

Even though it might seem that the travel agency market is experiencing severe problems, there are also a few opportunities that might help the agencies to make it through these troubled times. Recent research that has been done regarding the travelling preferences of the population has led to the conclusion that three new destinations getting more and more attention. Each of these three destinations assumes a different combination of prospective tourists and amount of commissions:

- Destination 1: small amount of prospective tourists, high commissions

- Destination 2: medium amount of prospective tourists, medium commissions

- Destination 3: large amount of prospective tourists, low commissions

Another opportunity for the travel agencies comes from research done within the country. The results of this study have revealed that there are three areas in the country that have shown an increase in the amount of money spent on travelling, by either travelling more often, longer or to more expensive destinations. In the heart of each area there is office space available, which can be rented by travel agencies interested in this opportunity. Also each office space has its own advantages and disadvantages:

- Office 1: city centre, high prospective customers, high competition, high costs

- Office 2: medium wealth area, low prospective customers, low competition, low costs

- Office 3: suburbs, medium prospective customers, low competition, medium costs, adjusted offering needed

As a direct consequence of the financial crisis, increase in unemployment, and higher inflation levels, several smaller tourism agencies have filed for bankruptcy. These smaller agencies have made it known that they are willing to sell whichever parts of their organisation that might interest other agencies (e.g. customer list, partner contacts, etc.). This can be seen as an opportunity by the three main agencies, which can purchase and use these offered resources to compensate their own weaknesses. The offerings of the small agencies are as follows:

- Agency 1: off-season travel packages

- Agency 2: customer list that represents a completely different market segment (retired people)

- Agency 3: list of partners that offer commissions for a destination that has a fairly constant number of travellers (between low and medium number)

Two major opportunities come from innovations that have been developed in the recent years. First of all, the innovations in the information and communication areas have brought a few new successful trends that any type of organisation can benefit from, such as social media. Second of all, the innovations done in the area of public transportation have helped produce faster, less costly aircrafts that can travel longer distances before they need to refuel, and can carry a larger number of passengers and cargo. What this means, for travel agencies, is that newer routes that are more direct, cheaper and faster might be offered by airlines. Also since there are more seats available, travel agencies can offer group deals on more routes and with more ease. Figure 2 shows an example of how these cards are structured.

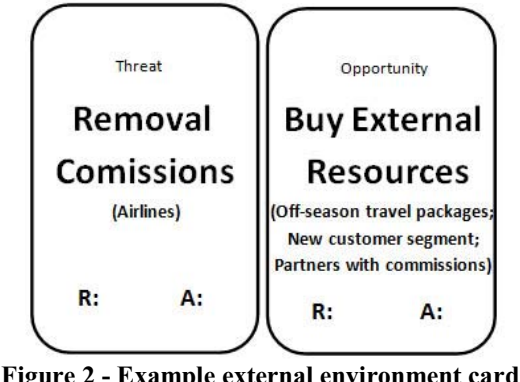

\section{Internal environment}

Each of the agencies has a similar internal environment with some remarkable differences that come from the way they have built their competitive position. In order to simplify the process of comparing the three agencies, all the canvases contain the same information. This information is rated by using stars, which can range from 0 to 3 . Zero stars means that the organisation does not have that specific feature and it is marked with a black star. For example, JourneyTime has loyalty programs as a defining feature, but none of the other two agencies have that feature implemented. Thus JourneyTime will have three stars at the loyalty programs feature in the Value proposition building block of its canvas, but the other two agencies will have a black star next to the loyalty programs (Figure 3 ). 


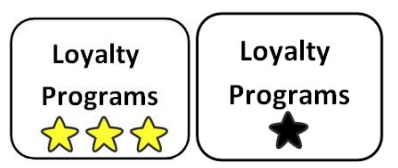

Figure 3 - Example internal environment cards

Environmental analysis

As a conclusion to the internal and external analyses, the participants are provided with a simplified SWOT analysis, which contains only the most relevant five environmental factors that have an influence on the three agencies. The choice of providing a simplified SWOT analysis is made in order to shorten the time spent on playing the game since it is only meant to be a demonstration of the method. Participants should learn and understand what the purpose of a SWOT analysis is and how they can use it as a starting point for the strategic planning process. The goal is to generate alternative strategies based on the information from the SWOT analysis, and to choose the most suitable one at a later point in time with the help of the QSPM. This completes the first two phases in the game where participants learn how to analyse and strategize the future of their organisation.

The event

After the second phase is completed, an unexpected global event will occur which will prompt the three teams to reassess the choices they have made in the previous phases. The event is triggered by a very popular touristic area being declared unsafe for tourism because of natural disaster. All three agencies rely heavily on that region for revenues, since $20 \%$ of their annual income comes from tickets or travel packages sold for that region. We have chosen to introduce an unexpected event to simulate the dynamic environment of organisations where you cannot assume that you can know or anticipate all the factors that can influence your organisation.

The projects

After the first two phases of the game are completed, the teams need to make the final decisions regarding their agencies. They need to decide which projects to implement in their organizations in order to support the chosen strategy, by spending a limited budget of 10 coins. Each project has an assigned cost and a benefit it will have after implementation (Figure 4). The projects are grouped in the nine building blocks of the BMC in order to better visualize where the improvements will have effect. We have chosen to limit the amount of resources (coins) each agency has to spend in order to stress the idea that decisions need to be made. Through this we familiarize the participants with the decision making process that takes place in organisations.

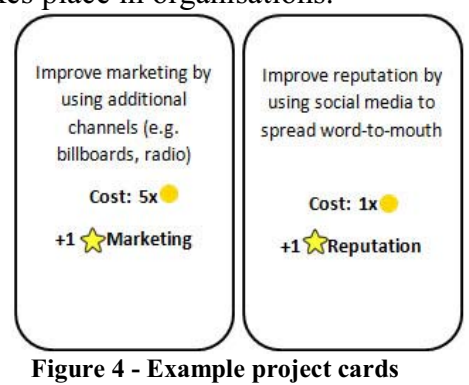

\section{Oucomes of the game}

At the end of the game each team should have a revised business model based on their chosen strategy and projects. The participants should learn how to think about the mission, vision an organisation, how to design its business model, how to take advantage of the environment and formulate strategies accordingly, and how to take the next step to make the chosen strategy achievable with objectives and projects. Besides this the participants should learn how to use the specific strategy models as means of communication, how to think when planning the future of an organisation, and how important it is to have an effective and efficient strategic planning process.

\section{Game testing}

The game has been tested on several occasions with a small group of students which has led to the version which I have currently presented. Improvements have been made in simplifying the game, reducing the steps, balancing the cards, and in the overall gaming session.

The current version has recently been tested on a group of 25 students from the University of Twente as part of one their courses. The students were notified about this session one day ahead, and were asked to read some information about the game and a summary about the strategy models. During the gaming session they were asked to formulate a mission, vision, and organisational values, to design their business model (assign the cards to the building blocks of a BMC), to identify/categorize the external forces (PEST analysis, Five Forces Framework), to formulate alternative strategies (Confrontation Matrix), to choose a strategy (QSPM), to formulate objectives (Strategy Map), and to choose projects.

As guidance, the students were provided with a game report that they could fill in. This report served a double purpose: it provided the students with clear guidelines to what they needed to do, and it could also be used to evaluate their results after the session. Besides the game report, the students were also asked to fill in a feedback form which used a 5-point Likert scale. The feedback form contained questions about the game, the underlying method, and the session.

From all the forms that were collected, 22 contained full responses, thus they were included in an analysis. Out of the three categories that were analysed, the underlying method received the best results. Varying between the questions, between $70-85 \%$ of the students gave average or above score to the following aspects of the underlying method: the strategy models were appropriate for the required tasks, the combination of strategy models provided a good overview of the whole strategic planning process, using a unified language (common concepts) improved the communication between team members, using this structured approach sped up the strategic planning process (efficiency), and with the help of the selected strategy models they were able to produce the expected results (effectiveness). Regarding the game, $72 \%$ of the students gave average or above scores to the game being considered a representation of a real life situation, while $90 \%$ considered the information provided about the strategy models to be helpful to them in the process of the game. In respect to the session, $70 \%$ of the students gave average or above scores to the usefulness of the instructions provided, and to enjoying playing the game. 
The areas in which the method scored lowest were the transition between strategy models which was not considered to be very easy, and they considered they were not able to make a clear plan for the future of the organisation. For the game, the lowest scores were noted for the amount of information regarding the organisation and its environment, the game cards, the process of the game, and the allotted time for the game. Regarding the session, the lowest scores were given for the instructions being understandable, the information from the session being useful, the structure of the session, and the information learned to be valuable for strategic planning.

It needs to be mentioned that none of the average scores for any of the questions were below 2.5 on a 5-point Likert scale. Also in the open feedback part of the form, the majority of comments were regarding the fact that the students didn't have enough time to prepare for the session, they didn't know what to expect, there was a lot of new information, and it lacked a competitive drive.

Table 1 shows the descriptive statistics of the feedback form for the 22 viable responses. The sum, mean and standard deviation per question and per category are included.

\begin{tabular}{|c|c|c|c|c|}
\hline & $\mathrm{N}$ & Sum & Mean & Std. Deviation \\
\hline Game_01 & 22 & 63 & 2.86 & .889 \\
\hline Game_02 & 22 & 58 & 2.64 & .953 \\
\hline Game_03 & 22 & 59 & 2.68 & .894 \\
\hline Game_04 & 22 & 57 & 2.59 & .959 \\
\hline Game_05 & 22 & 74 & 3.36 & .727 \\
\hline Game_06 & 22 & 56 & 2.55 & .912 \\
\hline Method_01a & 22 & 70 & 3.18 & .907 \\
\hline Method_01b & 22 & 66 & 3.00 & .756 \\
\hline Method_01c & 22 & 70 & 3.18 & .907 \\
\hline Method_01d & 22 & 68 & 3.09 & .750 \\
\hline Method_02 & 22 & 60 & 2.73 & .827 \\
\hline Method_03 & 22 & 68 & 3.09 & 1.151 \\
\hline Method_04 & 22 & 70 & 3.18 & 1.220 \\
\hline Method_05 & 22 & 66 & 3.00 & 1.024 \\
\hline Method_06 & 22 & 63 & 2.86 & .834 \\
\hline Method_07 & 22 & 57 & 2.59 & 1.221 \\
\hline Session_01 & 22 & 55 & 2.50 & .913 \\
\hline Session_02 & 22 & 63 & 2.86 & .834 \\
\hline Session_03 & 22 & 59 & 2.68 & 1.171 \\
\hline Session_04 & 22 & 56 & 2.55 & 1.143 \\
\hline Session_05 & 22 & 58 & 2.64 & 1.217 \\
\hline Session_06 & 22 & 64 & 2.91 & 1.192 \\
\hline Game & 22 & 61.167 & 2.7803 & 0.91896 \\
\hline method & 22 & 65.8 & 2.99091 & 0.97452 \\
\hline Session & 22 & 59.167 & 2.6894 & 1.07811 \\
\hline
\end{tabular}

The game reports revealed that two of the six groups managed to successfully fulfil all the tasks of the game and propose a well-defined strategy accompanied by good organisational objectives and projects. The other teams had the most difficulties with defining the mission, vision and values of their organisations, and with defining the right objectives.

\section{DISCUSSION AND LIMITATIONS}

One of the main aspects that need to be discussed is the use of students for testing the game. We have chosen to do this for several reasons. First of all, we wanted to test the game with a group of participants that we could easily have access to and that was willing to participate in a beta version of the game that might not be complete or well balanced. Usually experienced practitioners do not have a lot of time available for this kind of testing and we did not want to exhaust our pool of potential candidates on a rough version of the game. Also we consider university students to have the competencies needed to identify if there are any major flaws in the game. Second of all, our intention was to use students to test the major mechanics in the game and see if they were well designed and intuitive enough. Usually students have experience with playing games on different platforms and can provide valuable information regarding game mechanics that are a bit rough or unrealistic. Also they can help determine if the universe of play is well represented in the game. Last but not least, the game should be able to be played by participants that do not know everything about strategic planning and is meant to be a learning experience. By using students, we can determine if the information we provide is sufficient for the learning experience and can help them complete the game. Having said all of this, the end goal is still to have the game tested in later stages with a group of experienced practitioners that can provides us with feedback based on their experience in organisations.

One of the main limitations of the game at the moment is that it still feels like an assignment instead of a game. Because of this the game feels a bit heave and complex. Based on the feedback from this first large scale test of the game there are several improvements that are going to be implemented before the next testing session: the game is going to be played competitively (each team can lose, maintain, or gain market share), each team member is going to have a predefined role with specific responsibilities and knowledge (team members will need to cooperate to have the best results), participants will have more liberty in the choices they can make, the use of the strategy models will be simplified, the game cards will be reworked, the time allotted for the game will be increased.

Another limitation is the use of students for game testing. Although they provide valuable feedback the game can only be considered finished after several rounds of testing with experienced practitioners. We are planning an arranging these sessions as soon as possible, both with the cooperative and the competitive version of the game which is being developed at the moment. 


\section{CONCLUSION}

In this paper we have briefly presented the method of [3] al. for the strategic planning process, which has led to the development of a new strategy-based serious game. Although the game is still under development and testing, we consider it to have promising potential for becoming a useful tool to educate future participants. The results from the first large scale test have revealed a series points of improvement which will be addressed in future work.

Developing a serious game is not an easy task and we have experienced most of the pitfalls of such a pursuit. The main challenge with the kind of game we have developed is keeping it playable and enjoyable whilst not sacrificing any of the steps that need to be included.

\section{REFERENCES}

[1] T. Susi, M. Johannesson and P. Backlund, "Serious Games - An Overview," Institutionen för kommunikation och information, Skovde, 2007.

[2] K. Corti, "Game-based learning; a serious business application," Informe de PixelLearning, vol. 6, no. 34, pp. 1-20, 2006.

[3] A. Aldea, M.E. Iacob, D. Quartel and H. Franken, "Strategic planning and Enterprise Architecture," Enterprise Systems Conference (ES) IEEE, no. November, pp. 1-8, 2013.

[4] K. Peffers, T. Tuunanen, M. Rothenberger and S. Chatterjee, "A design science research methodology for information systems research," Journal of Management Information Systems, vol. 24, no. 3, pp. 45-77, 2008.

[5] G.A. Gunter, R.F. Kenny and E.H. Vick, "A case for a formal design paradigm for serious games," The Journal of the International Digital Media and Arts Association, vol. 3, no. 1, pp. 93-105, 2006.

[6] C. Harteveld, Triadic game design, Heidelberg: Springer, 2010.

[7] S. Deterding, D. Dixon, R. Khaled and L. Nacke, "From game design elements to gamefulness: defining gamification," Proceedings of the 15th International Academic MindTrek Conference: Envisioning Future Media Environments, no. ACM, pp. 9-15, 2011.

[8] A. Yusoff, R. Crowder, L. Gilbert and G. Willis, "A conceptual framework for serious games," Ninth IEEE International Conference on Advanced Learning Technologies, pp. 21-23, 2009.

[9] R. Rouse III, Game design theory and practice, Plano, TX: Wordware Publishing Inc., 2001.

[10] C.W. Hill and G.R. Jones, Strategic management: an integrated approach, Boston: Cengage Learning, 2008.

[11] J.D. Hunger and T.L. Wheelen, Essentials of strategic management, New Jersey: Prentice Hall, 2003.

[12] C.D. Scott, D.T. Jaffe and G.R. Tobe, Organisational vision, values and mission, Menlo Park: Crisp Publications, 1993.
[13] Object Management Group, "BMM 1.1," 2010. [Online]. Available: http://www.omg.org/spec/BMM/1.1/.

[14] A. Campbell and S. Yeung, "Brief case: mission, vision and strategic intent," Long Range Planning, vol. 24, no. 4, pp. 145-147, 1991.

[15] A. Osterwalder and Y. Pigneur, Business model generation: a hanbook for visionaries, game changers, and challengers, Hoboken, NJ: John Wiley \& Sons, 2010.

[16] W. Perreault and J. McCarthy, Basic marketing: a globalmanagerial approach, New York: McGraw-Hill/Irwin, 2002.

[17] J. Barney, "Firm resources and sustained competitive advantage," Journal of Management, pp. 99-120, 1991.

[18] D. Teece and G. Pisano, "The dynamic capabilities of firms: an introduction," Industrial and Corporate Change, vol. 3, pp. 537-556, 1994.

[19] I. Britton and C. Worthington, The business environment, Harlow: Financial Times / Pearson Education, 2006.

[20] M. Porter, Competitive strategy: techniques for analysing industries and competitors, New York: Free Press, 1980.

[21] R. Griffin, Fundamentals of management, Mason, OH: Cengage Learning, 2011.

[22] W.C. Kim and R. Mauborgne, Blue ocean strategy: how to create uncontested market space and make competition irrelevant, Boston: Harvard Business School Press, 2005.

[23] H. Weihrich, "The TOWS matrix - a tool for situational analysis," Long Range Planning, vol. 15, no. 2, pp. 5466, 1982.

[24] F. David, "The strategic planning matrix - a quantitative approach," Long Range Planning, vol. 19, no. 5, pp. 102107, 1986.

[25] R.S. Kaplan and D.P. Norton, Strategy maps: converting intangible assets into tangible outcomes, Boston: Harvard Business School Press, 2004.

[26] R.S. Kaplan and D.P. Norton, "The balanced scorecard measures that drive performance," Harvard Business Review, Vols. January-February, pp. 71-79, 1992.

[27] M.E. Iacob, H. Jonkers, D. Quartel, H. Franken and H. van den Berg, Delivering enterprise architecture with TOGAF and ArchiMate, Van Haren Publishing, 2012.

[28] J. Ward, E. Daniel and J. Peppard, "Building better business cases for IT investments," California Management Review, 2007.

[29] M. Treacy and F. Wiersema, "Customer intimacy and other value disciplines," Harvard Business Review, vol. 71, no. 1, pp. 84-93, 1993.

[30] M.E. Iacob, H. Jonkers, M. Lankhorst, E. Proper, Dick A.C. Quartel, ArchiMate 2.0 Specification, The Open Group, Van Haren Publishing, February 2012, 1-93721800-3. 